\title{
Immune effects against influenza $A$ virus and a novel DNA vaccine with co-expression of haemagglutinin- and neuraminidase-encoding genes
}

\author{
Correspondence \\ Mingyuan Li \\ zxllmyus@yahoo.com
}

Received 23 September 2008 Accepted 8 March 2009

\author{
Weidong Zhang, ${ }^{1,2}+$ Wanyi $\mathrm{Li}^{1}{ }^{1}+$ Yan $\mathrm{Li}^{3}{ }^{3}$ Hong Li, ${ }^{1}$ Baoning Wang, ${ }^{1}$ \\ Fengping Wang, ${ }^{1}$ Yuanjun Zhu, ${ }^{1}$ Zhonghua Jiang, ${ }^{1}$ Li Zhong ${ }^{1}$ \\ and Mingyuan $\mathrm{Li}^{1,3}$ \\ ${ }^{1}$ West China School of Preclinical and Forensic Medicine, Sichuan University, Chengdu, Sichuan \\ 610041, PR China \\ ${ }^{2}$ Department of Pathobiology, Basic Medical School of Hebei Medical University, Hebei, PR China \\ ${ }^{3}$ State Key Laboratory of Oral Diseases, Sichuan University, Chengdu, Sichuan 610041, PR China
}

\begin{abstract}
The high variability of influenza virus causes difficulties in the control and prevention of influenza, thus seeking a promising approach for dealing with these problems is a hot topic. Haemagglutinin (HA) and neuraminidase (NA) are major surface antigens of the influenza virus, and provide effective protection against lethal challenges with this virus. We constructed a DNA vaccine (pHA-IRES2-NA) that co-expressed both HA and NA, and compared its protective efficacy and immunogenic ability with that of singly expressed $\mathrm{HA}$ or NA, or a mixture of the two singly expressed proteins. Our findings showed that both HA and NA proteins expressed by pHAIRES2-NA could be detected in vivo and in vitro. The protection of DNA vaccines was evaluated by serum antibody titres, residual lung virus titres and survival rates of the mice. In the murine model, immunization of pHA-IRES2-NA generated significant anti-HA and anti-NA antibody, increased the percentage of $\mathrm{CD}^{+}$cells and gamma interferon-producing $\mathrm{CD}^{+}$cells and the ratio of Th1/Th2 (T helper) cells, which was comparable to the effects of immunization with $H A$ or NA DNA alone or with a mixture of HA and NA DNA. All the mice inoculated by pHA-IRES2-NA resisted the lethal challenge by homologous influenza virus and survived with low lung virus titre. In addition, previous studies reported that co-expression allowed higher-frequency transduction compared to co-transduction of separated vector systems encoding different genes. The novel $\mathrm{HA}$ and NA co-expression DNA vaccine is a successful alternative to using a mixture of purified $\mathrm{HA}$ and NA proteins or HA and NA DNA.
\end{abstract}

\section{INTRODUCTION}

Influenza virus is the pathogenic agent of influenza, which occurs as numerous localized outbreaks or regional epidemics and occasionally as a global pandemic. The severity of influenza epidemics is a formidable publichealth challenge as well as a significant socio-economic burden. Haemagglutinin (HA) and neuraminidase (NA) are two major antigens on the surface of influenza virus. The most commonly used vaccines against the influenza

†These authors contributed equally to this paper.

Abbreviations: ConA, concanavalin A; CTL, cytotoxic T lymphocyte; HA, haemagglutinin; $\mathrm{HI}$, haemagglutinin inhibition; IFN- $\gamma$, gamma interferon; $\mathrm{IL}$, interleukin; NA, neuraminidase; $\mathrm{NI}$, neuraminidase inhibition; $\mathrm{PE}$, phycoerythrin; SI, stimulation index; $\mathrm{TCID}_{50}, 50 \%$ tissue culture infection dose; Tc cell, cytotoxic T cell; Th cell, T helper cell. virus comprise purified preparations of HA and NA, and are designed to induce anti-HA and anti-NA antibodies (Lambkin et al., 2004). Antibodies against HA and NA are major dependent elements that can protect a host against the influenza virus (Bona et al., 2004). Anti-HA antibody prevents a virus from binding to the sialic acid receptor of a host cell and the fusion of a virion with the plasma membrane, and anti-NA antibody prevents the spreading of the virus among cells by inhibiting the enzymic activity of NA and reduces morbidity in vivo (Johansson et al., 1993).

The high variability of influenza virus causes difficulties in the control and prevention of influenza. Thus, seeking a promising approach for dealing with these problems is a hot subject, to which researchers from different countries persistently pay attention. DNA vaccine has become the 
focus of widespread interest, not only because of its novelty and simplicity, but also because of its efficacy in inducing specific immunity (Donnelly et al., 1997; Tighe et al., 1998). The National Institutes of Health of the USA and the World Health Organization have recommended an emphasis on developing an influenza DNA vaccine (Gruber, 2002; Ulmer, 2002). Previous studies have confirmed that an influenza DNA vaccine encoding HA induced specific and strong immune responses, and provided protection against influenza virus challenge in animals (Bot et al., 1997; Johnson et al., 2000). Meanwhile, the NA-encoding gene is a strong and potent candidate for a DNA vaccine for influenza. Chen et al. (2005) reported that NA DNA vaccine completely protected various strains of mouse against homologous or heterologous virus attack in $\mathrm{BALB} / \mathrm{c}$ mice. Therefore, several scientists suggested that a vaccine based on NA could provide more effective protection against the influenza virus in combination with a classic HA-based vaccine (Fiers et al., 2001; Li et al., 2006).

In the development of DNA vaccines, one of the major obstacles is low transduction and expression. Shen et al. (2000) found that co-expression and co-transduction of multiple genes are important in the investigation of gene therapy and infectious disease prophylaxis, because coexpression vectors can improve the efficacy of transduction and expression. Thus, our objectives for this study were: (1) to construct a DNA vaccine for co-expression of HA and NA, and (2) to evaluate its immune efficiency by comparing results from different DNA vaccines involving single expression of either HA or NA, a mixture of the two single expressions, and a co-expression of HA and NA.

\section{METHODS}

Eukaryotic expression plasmid construction. pcDNA3.1 $(+) / \mathrm{HA}$ and pcDNA3.1 $(+)$ /NA were constructed by inserting the products of $H A$ and NA RT-PCR cloned from the influenza A virus (strain A/PR/ $8 / 34, \mathrm{H} 1 \mathrm{~N} 1$ ) as described previously (Zhang et al., 2006). pIRES2EGFP (Clontech) was modified by replacing the EGFP (enhanced green fluorescent protein)-encoding fragment with a NotI/BstXI digested and blunted NA fragment to produce vector pIRES2-NA. The HA fragment was inserted into the BglII and NheI cloning sites of pIRES2-NA to construct the novel recombinant plasmid pHA-IRES2NA. All inserted fragments were confirmed by restriction endonuclease digestion analysis, PCR amplification and sequencing using an ABI Prism 377XL DNA sequencer. pcDNA3.1 $(+) / \mathrm{HA}$, pcDNA3.1 $(+) / \mathrm{NA}$ and pHA-IRES2-NA DNAs were extracted and purified on a large scale using the EndoFree plasmid maxi kit (Qiagen).

Cell transfection assay. Purified eukaryotic expressing plasmids DNAs of pcDNA3.1 $(+) / \mathrm{HA}$, pcDNA3.1 $(+) / \mathrm{NA}$ and pHA-IRES2NA were used to transfect HEK293 cells by PolyFect transfection reagent (Qiagen). The preparation and culture of HEK293 cells were carried out as described previously (Zhang et al., 2006). The transfection procedures were performed according to the reagent manufacturer's instructions. After transfection for $48 \mathrm{~h}$, the cells were washed with PBS and fixed by $4 \%$ paraformaldehyde. Goat serum was used to block the blank, then the cells were incubated with rabbit
anti-A/PR/8/34(H1N1) (prepared by our laboratory and stored at low temperature) serum or mouse antibodies against HA and NA (a kind gift from Lanzhou Institute of Biological Products, Lanzhou, PR China), followed by fluorescein-conjugated AffiniPure goat antirabbit IgG or goat anti-mouse IgG (Beijing ZhongShan Golden Bridge Biotechology). Finally, the expression of HA and NA proteins was assessed by fluorescence microscopy.

Expression of eukaryotic expression plasmids in muscle. Mice were inoculated with $50 \mu \mathrm{g}$ purified plasmid DNA by intramuscular injection at the right quadriceps. At 4, 8, 12, 24, 36, 48 and $72 \mathrm{~h}$ after inoculation, the inoculated muscle tissues were excised from the euthanized mice, then fixed in $4 \%$ paraformaldehyde overnight. After being dehydrated by a series of ethanol solutions and cleared in xylene, the fixed muscle samples were embedded in molten paraffin wax, which were then solidified by cooling so that they could be sliced into $5 \mu \mathrm{m}$ sections using a microtome, mounted on glass slides and dried. These sections were dewaxed in xylene and dehydrated through a series of ethanol and PBS solutions, then incubated in $3 \% \mathrm{H}_{2} \mathrm{O}_{2}$ for 5-10 $\mathrm{min}$ at room temperature to block endogenous peroxidase. Sections were then blocked with 5 or $10 \%$ goat serum for $30 \mathrm{~min}$. The appropriate diluted anti-HA or anti-NA antibody was added and the sections were incubated at $4{ }^{\circ} \mathrm{C}$ overnight. After washing, biotinlabelled goat anti-mouse antibody (Boster Biotechnological) was applied. Following a $45 \mathrm{~min}$ incubation at room temperature and washing, streptavidin-peroxidase was added. The sections were incubated in 3,3-diaminobenzidine substrate for $5 \mathrm{~min}$, and then counterstained with haematoxylin. The slides were dehydrated by a series of ethanol solutions, placed in xylene, and mounted with a coverslip using DPX resin medium. The results were checked with a microscope.

Immunization and virus challenge. Four- to six-week-old female $\mathrm{BALB} / \mathrm{c}$ mice were obtained from the animal care centre of Sichuan University and bred under pathogen-free conditions. All experiments were done with institutional approval. A total of 60 mice were divided into 6 groups, with 10 mice in each group. Each group of mice wa injected separately with one of the following: pHA-IRES2$\mathrm{NA}, \quad \operatorname{pcDNA} 3.1(+) / \mathrm{HA}+\mathrm{pcDNA} 3.1(+) / \mathrm{NA} \quad(\mathrm{HA}+\mathrm{NA} \quad \mathrm{DNA})$, pcDNA3.1 $(+) / \mathrm{HA}$ (HA DNA), pcDNA3.1(+)/NA (NA DNA), pcDNA3.1 $(+)$ or PBS. The mice were immunized by intramuscular injection of the quadriceps of both legs $(50 \mu \mathrm{g}$ in $50 \mu \mathrm{l}$ PBS for each leg) twice with a 3 week interval between injections.

Seven days after the booster immunization, the mice were challenged with influenza virus, the mouse-adapted strain, A/PR/8/34(H1N1) $\left[40 \times \mathrm{LD}_{50}\right]$ by intranasal administration with $20 \mu \mathrm{l}$ viral suspension under light anaesthetization. This infection caused rapid and widespread viral replication in the lungs and death of the unimmunized mice in 6-8 days (Chen et al., 2005; Tamura et al., 1992a).

Specimen collection. The collection was performed according to the method reported by Chen et al. (2005) and Tamura et al. (1992b). The first immunized sera were collected from each animal of every group through the tail bleeding 1 day before the booster immunization. The second immunized sera were collected from four randomly chosen mice by heart bleed under anaesthetization with chloroform 3 days after the virus challenge. A ventral incision along the median line from the xiphoid process to the point of the chin was made for each mouse. The trachea and lungs were taken out and washed twice with $1 \mathrm{ml}$ PBS containing $0.1 \%$ BSA. The bronchoalveolar lavage fluids were centrifuged to remove cellular debris and used for virus titration.

Splenocyte proliferation response. For splenocyte proliferation assays, the spleens from the six groups of mice were processed as follows: in a 96-well flat-bottom plate, the splenocytes $\left(5 \times 10^{5}\right.$ cells 
per well) were stimulated with $0.5 \mu \mathrm{g}$ concanavalin A (ConA), $1.25 \mu \mathrm{g}$ recombinant $\mathrm{HA}$ or NA separately. After $48 \mathrm{~h}$ incubation with $5 \%$ $\mathrm{CO}_{2}$ at $37^{\circ} \mathrm{C}, 20 \mu \mathrm{l}$ MTT [3-(4,5-dimethylthiazol-2-yl)-2,5-diphenyltetrazolium bromide] per well was added and incubated for another $4 \mathrm{~h}$. The plate was centrifuged at $1000 \mathrm{~g}$ for $10 \mathrm{~min}$ and the supernatants were aspirated gently and discarded. In order to dissolve the formazan salt crystals, $100 \mu \mathrm{l}$ DMSO was added into each well and incubated for $30 \mathrm{~min}$ at $37{ }^{\circ} \mathrm{C}$. The absorbance at $550 \mathrm{~nm}$ was then measured. The results were indicated as a stimulation index (SI): $A_{550}$ of the experiments divided by $A_{550}$ of the pcDNA3.1 $(+)$ control.

Antibody assay. The HA inhibition (HI) assay was carried out as follows. A total of $25 \mu \mathrm{l} 4 \mathrm{HA}$ units virus (A/PR/8/34, H1N1) per well was added to a 96-well microtitre plate containing $25 \mu \mathrm{l}$ sera twofold serially diluted with PBS. After being mixed and incubated at $4{ }^{\circ} \mathrm{C}$ for $1 \mathrm{~h}, 50 \mu \mathrm{l} 0.5 \%(\mathrm{v} / \mathrm{v})$ chicken erythrocytes was added to each well and the plate was incubated at $4{ }^{\circ} \mathrm{C}$ for $30-45 \mathrm{~min}$. The HI end-point titres were determined as the reciprocal of the highest serum dilution that completely inhibited haemagglutination.

The NA inhibition (NI) test comprised two parts: an NA activity assay and a self test of NI. Allantoic fluid containing influenza virus was twofold serially diluted in $0.15 \mathrm{M}$ saline. A total of $20 \mu \mathrm{l}$ of the virus dilution or control was transferred to each of a series of Eppendorf tubes and $20 \mu \mathrm{l}$ fetuin reagent was added. After the solution was mixed well, it was stood at $37{ }^{\circ} \mathrm{C}$ for $18 \mathrm{~h}$ and was then cooled to $20{ }^{\circ} \mathrm{C}: 20 \mu \mathrm{l}$ periodate reagent was added, mixed thoroughly and allowed to incubate at room temperature for exactly $20 \mathrm{~min}$. A brown colour formed after addition of $200 \mu \mathrm{l}$ arsenite reagent and gradually disappeared with shaking of the tube. A total of $500 \mu$ l thiobarbituric acid reagent was added and the reaction mixture was boiled in a water bath for $15 \mathrm{~min}$. The tube was cooled to room temperature in ice water and $800 \mu \mathrm{l}$ butanol reagent added. The extracted red colour was passed into an organic phase by vigorously shaking the tube by hand. The upper phase was pipetted into a colorimeter cuvette after centrifuging the tube at 1500 r.p.m. for 5 min (Eppendorf $5415 \mathrm{D}$ centrifuge, F-45-2411 rotor). The fetuin blank was used to equilibrate the spectrometer, and the absorbance of the tested sample was read against a blank at $549 \mathrm{~nm}$. The virus concentration gave an absorbance of 0.5 , which was determined as 1 enzyme unit to be used in the NI test. The virus suspension was adjusted to the concentration representing 1 enzyme unit. A total of $50 \mu \mathrm{l}$ adjusted virus suspension was mixed with $50 \mu \mathrm{l}$ of the tested serum dilution at $0.5 \log _{10}$ dilution (1:3.2 dilution approximately). After incubating at $37^{\circ} \mathrm{C}$ for $1 \mathrm{~h}, 100 \mu \mathrm{l}$ fetuin was added and the reaction carried out at $37{ }^{\circ} \mathrm{C}$ for $18 \mathrm{~h}$ in a water bath. The remaining part of the procedure was repeated as described for the NA activity assay. The NI titre of the antiserum was defined as the dilution of serum giving $50 \%$ inhibition of NA activity (Aymard-Henry et al., 1973).

Influenza virus titrations. Madin-Darby canine kidney cells were cultured in 24-well plates and formed a confluent monolayer. The bronchoalveolar lavage fluid was 10-fold serially diluted from $1: 10$ and inoculated on Madin-Darby canine kidney cells: four wells of every concentration were repeated. After incubating at $37{ }^{\circ} \mathrm{C}$ for 2 days, the cytopathic effect was examined. The virus titre of each specimen, recorded as the $50 \%$ tissue culture infection dose $\left(\mathrm{TCID}_{50}\right)$, was calculated by the Reed-Muench method (Reed \& Muench, 1938). The virus titre in each group was expressed as the mean \pm SD of all specimens in each group.

Flow cytometry. The spleens of four BALB/c mice obtained from 3 days after the virus challenge were gently pressed through stainless steel mesh and the mononuclear cells were purified by densitygradient centrifugation through NycoPrep 1.077A (Axis-shield). The cells were adjusted to $5 \times 10^{6} \mathrm{cells} \mathrm{ml}^{-1}$ and maintained in RPMI1640 supplemented with $20 \%(\mathrm{v} / \mathrm{v})$ fetal calf serum in 6-well plates in the presence of phorbol-12-myristate-13-acetate (PMA; Sigma) $\left(100 \mathrm{ng} \mathrm{ml}^{-1}\right), 3 \mu \mathrm{M}$ monensin (Sigma) and $1 \mu \mathrm{g}$ ionomycin $\mathrm{ml}^{-1}$ (Sigma) overnight at $37^{\circ} \mathrm{C}$ in a humidified atmosphere containing $5 \% \mathrm{CO}_{2}$ (Alheim et al., 2001). Direct staining of the cultured cells was carried out as follows: FITC-labelled anti-mouse CD3 antibody and phycoerythrin (PE)-Cy5 labelled anti-mouse CD8 antibody (BD Pharmingen) were reacted with the cells for $20 \mathrm{~min}$ at room temperature in the dark. Then the cells were washed with PBS (0.01 M, pH 7.4) and fixed with $4 \%$ paraformaldehyde. After removal of the fixation fluid by centrifugation, the cells were permeabilized with $0.5 \%$ saponin for $15 \mathrm{~min}$ at RT. PE-labelled anti-mouse gamma interferon (IFN- $\gamma$ ) antibody (BD Pharmingen), PE-labelled anti-mouse interleukin 4 (IL-4) antibody (BD Pharmingen) or an isotype control antibody (BD Pharmingen) were added separately to the cells and incubated for $30 \mathrm{~min}$ at $4{ }^{\circ} \mathrm{C}$ in the dark. Cells were then resuspended in $500 \mu \mathrm{l}$ PBS and subjected to flow cytometric analysis. Data analysis was carried out using BD FACSAria software.

Specific cellular response of co-expression plasmid to HA and NA antigens. The splenocytes of BALB/c mice were obtained from vaccinated pHA-IRES2-NA and pcDNA3.1 $(+)$ mice. The cells were adjusted to $5 \times 10^{6}$ cells ml $\mathrm{ml}^{-1}$ and maintained in RPMI- 1640 supplemented with $20 \%(\mathrm{v} / \mathrm{v})$ fetal calf serum in 6-well plates in presence of $1.25 \mu \mathrm{g}$ recombinant $\mathrm{HA}$ or NA for $48 \mathrm{~h}$ at $37{ }^{\circ} \mathrm{C}$ in humidified atmosphere containing $5 \% \mathrm{CO}_{2}$. Other procedures were as described for the flow cytometry. The results were shown as the percentage of $\mathrm{CD}^{+}{ }^{+} \mathrm{CD} 8^{-}$and $\mathrm{CD} 3^{+} \mathrm{CD}{ }^{+}$cells, and the ratio of $\mathrm{IFN}-\gamma^{+} \mathrm{CD} 3^{+} \mathrm{CD} 8^{-} / \mathrm{IL}_{-} 4^{+} \mathrm{CD}^{-}{ }^{-} \mathrm{CD} 8^{-}$cells.

Statistical analysis. Data have been presented in the text and figures as means $\pm \mathrm{SD}$, with $N$ indicating the number of individual mice tested in the set of experiments. Group-group comparison data were analysed using one-way ANOVA test. A value of $P<0.05$ was considered significant.

\section{RESULTS}

\section{Transfection and expression of HA and/or NA plasmids into eukaryotic cells}

pHA-IRES2-NA, pcDNA3.1(+)/HA and pcDNA3.1 $(+) /$ NA were successfully used to transfect HEK293 cells. Stronger green fluorescent signals were clearly seen in the cells transfected by pHA-IRES2-NA, pcDNA3.1 $(+) / \mathrm{HA}$ and pcDNA3.1 $(+) / \mathrm{NA}$ (Fig. 1). It was shown that either $H A$ or NA genes could be transiently expressed in HEK 293 cells.

\section{Expression of co- and single-expression HA and/ or NA plasmids in muscle}

The in vitro experimental results confirmed that pHAIRES2-NA, pcDNA3.1 $(+) / \mathrm{HA}$ and $\operatorname{pcDNA3.1}(+) / \mathrm{NA}$ could be effectively expressed by eukaryotic cells. Therefore, these plasmids could be used as a DNA vaccine by intramuscular injection for preventing influenza. As shown in Fig. 2, there was no protein expression detected in the sections of muscle tissues of the controls inoculated with empty plasmid or PBS. However, HA or NA protein could be detected in the sections inoculated with pHAIRES2-NA, pcDNA3.1 $(+) / \mathrm{HA}$ and pcDNA3.1 $(+) / \mathrm{NA}$ by probing with anti-HA and /or anti-NA antibodies. The 

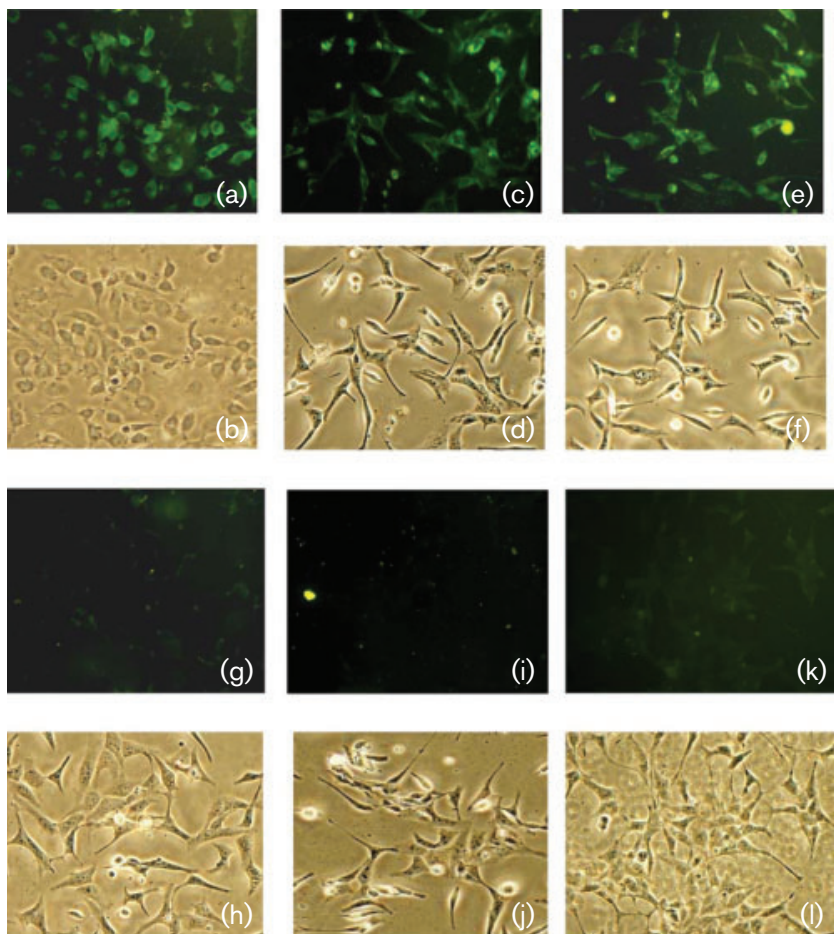

Fig. 1. Expression of HA and/or NA in HEK293 cells. HEK293 cells were transfected by plasmid DNAs encoding co- and singleexpression $\mathrm{HA}$ and/or NA, and expression was evaluated by indirect immunofluorescence. ( $a, b)$ Cells transfected with pcDNA3.1 $(+) / N A ;(c, d)$ cells transfected with pHA-IRES2-NA, incubated with antibody to $\mathrm{HA}$; $(e, f)$ cells transfected with $\mathrm{pHA}$ IRES2-NA, incubated with antibody to NA; $(g, h)$ cells transfected with empty plasmid; $(i, j)$ control of normal goat serum; $(k, 1)$ untransfected cells incubated with antibody to HA. Fluorescent images $(a, c, e, g, i, k)$ and phase contrast images $(b, d, f, h, j, l)$ are shown.

targeted proteins were detected as early as $8 \mathrm{~h}$ (data not shown) after the injection of the plasmid DNAs into the muscles, and could be detected $72 \mathrm{~h}$ after the transfer. The expression of targeted protein in vivo was observed by immunohistochemical staining.

\section{Splenocyte proliferation response to specific antigen}

The splenocytes from the vaccinated HA and NA groups proliferated at a significantly higher rate than their respective controls when stimulated in vitro with HA and NA antigen (Fig. 3). However, HA- and NA-driven proliferation of splenocytes was markedly lower when compared to the stimulation induced by ConA (data not shown). Specifically, the splenocytes from the HA + NA DNA vaccinated group exhibited a threefold to fourfold higher proliferation compared to their controls $(P<0.05)$. The response from the pHA-IRES2-NA vaccinated group was close to that of HA or NA single vaccinated group.
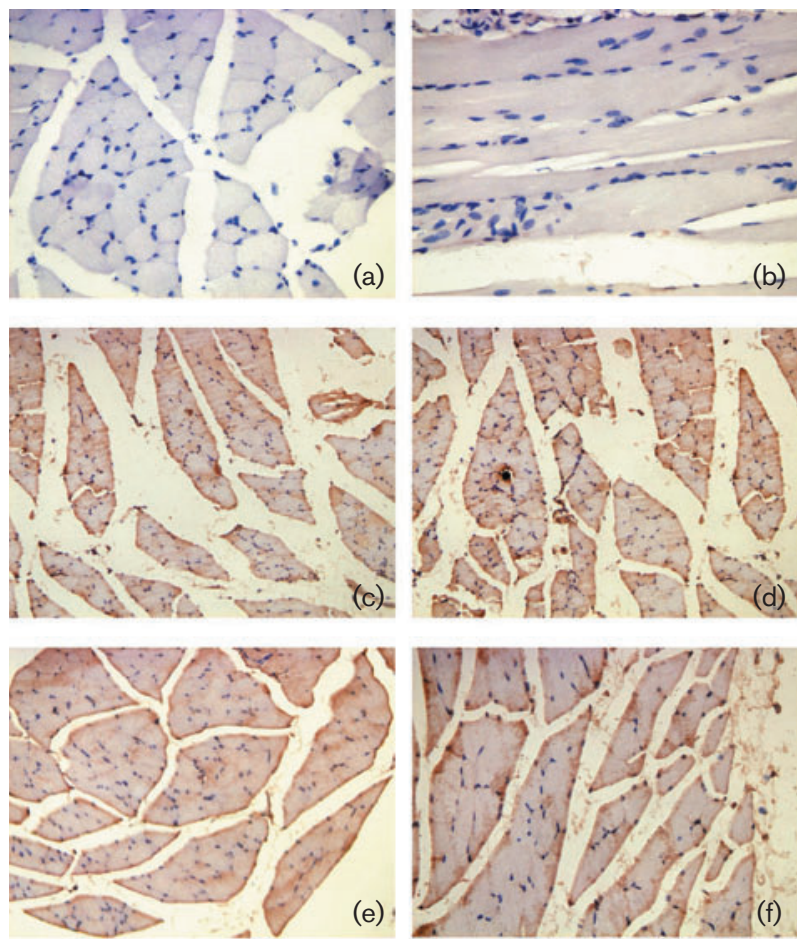

Fig. 2. Expression of $H A$ and/or $N A$ in mouse muscle at $72 \mathrm{~h}$ after the gene transduction. pcDNA3.1(+)/HA, pcDNA3.1(+)/NA and pHA-IRES2-NA were injected into the muscle tissue separately and the expression of $\mathrm{HA}$ or NA protein was detected by immunohistochemical staining with anti-HA (c, e) or anti-NA (d, f) antibody. $(a, b)$ Representative experiment after injection of PBS (a) and pcDNA3.1 $(+)(b)$; (c, d) representative experiment after injection of pHA-IRES2-NA and reaction with anti-HA (c) or antiNA (d); (e, f) representative experiment after injection of pcDNA3.1(+)/HA (e) and pcDNA3.1(+)/NA (f).

\section{Protection against A/PR/8/34(H1N1) challenge induced by co-expression of $H A$ and NA DNA in comparison with single expression of $H A$ or $N A$ DNA and a mixture of $H A$ and NA DNA}

The female BALB/c mice aged 4-6 weeks were divided into six groups. Of the six groups, three groups were immunized with $100 \mu \mathrm{g}$ pHA-IRES2-NA, or pcDNA3.1 $(+) / \mathrm{HA}$ or pcDNA3.1 $(+) / \mathrm{NA}$; one group was immunized with $100 \mu \mathrm{g}$ of a mixture of pcDNA3.1 $(+) / \mathrm{HA}$ and $\operatorname{pcDNA3.1}(+) / \mathrm{NA}$; and the other two groups were injected with empty plasmid or PBS as negative or blank controls, respectively. On the seventh day after booster immunization, the mice were challenged with a lethal dose $\left[40 \times \mathrm{LD}_{50}\right]$ of $\mathrm{A} / \mathrm{PR} / 8 / 34(\mathrm{H} 1 \mathrm{~N} 1)$ by intranasal drip. On the third day after the challenge, four mice from each group were sacrificed for the evaluation test of lung virus titres. The rest of the mice were observed for 3 weeks to calculate the survival rate of each group. The groups with pHAIRES2-NA and the mixture of HA and NA DNA immunization showed complete protective ability against 


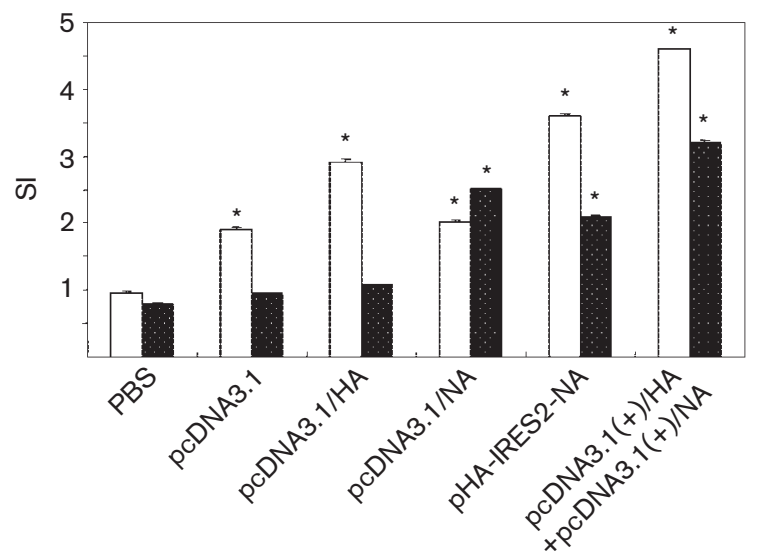

Fig. 3. Splenocyte proliferation induced by HA and NA after $48 \mathrm{~h}$ of in vitro culture. The splenocyte proliferation induced by $\mathrm{HA}$ in the pHA-IRES2-NA vaccinated group was close to that of the single $H A$ DNA vaccinated group and the HA + NA DNA group. However, the response induced by NA in the pHA-IRES2-NA vaccinated group was slightly lower than that of the single NA DNA vaccinated group and the HA+NA DNA group. The splenocyte proliferation induced in the HA+NA DNA vaccinated groups was significantly higher than that of respective controls when stimulated in vitro with $\mathrm{HA}$ and NA antigens. However, splenocyte proliferation induced by HA or NA was markedly lower when compared to the proliferation induced by ConA (data not shown). Specifically, the SI from the $\mathrm{HA}+\mathrm{NA}$ DNA vaccinated group exhibited a threefold to fourfold higher response compared to the pcDNA3.1 $(+)$ control $(P<0.05)$. The SI from the pHA-IRES2-NA vaccinated group was close to that of the HA DNA or NA DNA vaccinated group. A $P$ value $<0.05$ compared with the PBS group is indicated by an asterisk. White bars, HA; black bars, NA.

the homologous virus attack. The group with singleexpression HA DNA or NA DNA immunization demonstrated an $83 \%$ protective rate. In contrast, nearly all of the mice of the two control groups died within 7 days after the challenge. The data showed decreased residual lung virus titres to a different extent in all of the groups immunized with DNA vaccines carrying the NA gene, especially the group immunized with the mixture of HA + NA DNA, which exhibited a significant decrease of lung virus titres compared with other groups. The residual lung virus titre in mice immunized with pHA-IRES2-NA obviously declined, but not as much as the mixture of $\mathrm{HA}+\mathrm{NA}$ DNA immunized group. In the two control and the singleexpression HA DNA immunized groups, the residual lung virus titres were up to or over $10^{5} \mathrm{TCID}_{50}$ (Table 1).

\section{Comparison of antibody titres in mice induced by co- and single-expression HA and/or NA DNA}

The female BALB/c mice were immunized twice with a 3 week interval with the novel constructed influenza DNA vaccines co-expression or single-expression HA and/or NA. Two groups were injected with PBS and an empty vector as controls. The primary- and booster-immunized sera were collected by tail vein bleed and heart bleed. The specific antibodies elicited by the influenza DNA vaccines against $\mathrm{HA}$ and/ or NA were determined by HI and NI assays. The titres of $\mathrm{HI}$ and NI were represented as group geometric means. As shown in Fig. 4, both the novel HA and NA DNA co-expression construct and the mixture of $\mathrm{HA}+\mathrm{NA}$ DNA succeeded in inducing $\mathrm{HI}$ and NI antibodies the same as singly expressed $H A$ or NA DNA did. In the primary response, the $\mathrm{HI}$ antibody titres were lower $(<10)$ and the NI antibody titres were lower $(<5)$ in the responding groups; in the booster response the $\mathrm{HI}$ antibody titres were twofold to threefold higher, and the NI antibody titres were quintuple the level of the primary response. In the $\mathrm{HI}$ assay, the antibody level induced by pHA-IRES2-NA was as high as other responding groups, such as pcDNA3.1 $(+) /$ $\mathrm{HA}$ and the mixture of pcDNA3.1 $(+) / \mathrm{HA}$ and pcDNA3.1 $(+) / \mathrm{NA}$. In the NI assay, the antibody levels induced by pHA-IRES2-NA and the mixture of pcDNA3.1 $(+) / \mathrm{HA}$ and $\mathrm{pcDNA3.1}(+) / \mathrm{NA}$ were lower than pcDNA3.1 $(+) / \mathrm{NA}$ alone, but there were no evident differences among them by statistical analysis. Compared

Table 1. Protection against lethal virus challenge in the tested mice

\begin{tabular}{|lcc|}
\hline \multirow{2}{*}{ Group } & \multicolumn{1}{c|}{ Protection against virus challenge } \\
\cline { 2 - 3 } & $\begin{array}{c}\text { Lung virus titre } \text { TCID }_{50} \\
(\boldsymbol{n}=\mathbf{4})\end{array}$ & $\begin{array}{c}\text { No. of surviving mice/no. of } \\
\text { experimental mice }(\boldsymbol{n}=\mathbf{6})\end{array}$ \\
\hline pHA-IRES2-NA & $4.06 \pm 0.16$ & $6 / 6$ \\
pcDNA3.1 $(+) / \mathrm{HA}+$ pcDNA3.1 $(+) / \mathrm{NA}$ & $3.38 \pm 0.24^{*}$ & $6 / 6$ \\
pcDNA3.1 $(+) / \mathrm{NA}$ & $3.81 \pm 0.12$ & $5 / 6$ \\
pcDNA3.1 $(+) / \mathrm{HA}$ & $5.19 \pm 0.19 \dagger$ & $5 / 6$ \\
pcDNA3.1 $(+)$ & $5.88 \pm 0.22 \dagger$ & $1 / 6 \ddagger$ \\
PBS & $6.19 \pm 0.02 \dagger$ & $0 / 6 \ddagger$ \\
\hline
\end{tabular}

${ }^{\star}$ The residual lung virus titre of the HA + NA DNA immunized group has a distinct difference compared with other groups $(P<0.05)$.

$\dagger$ The residual lung virus titres of the three groups were higher than any of the other groups $(P<0.05)$.

¥The survival rates of the two groups were minimal compared to the other groups $(P<0.05)$. 
(a)

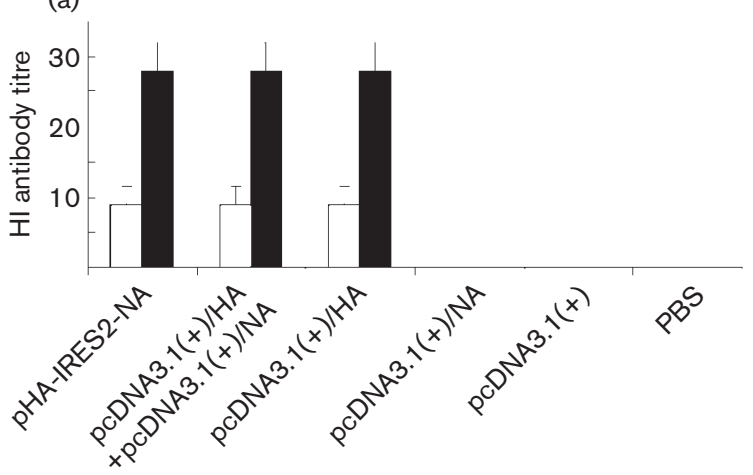

(b)

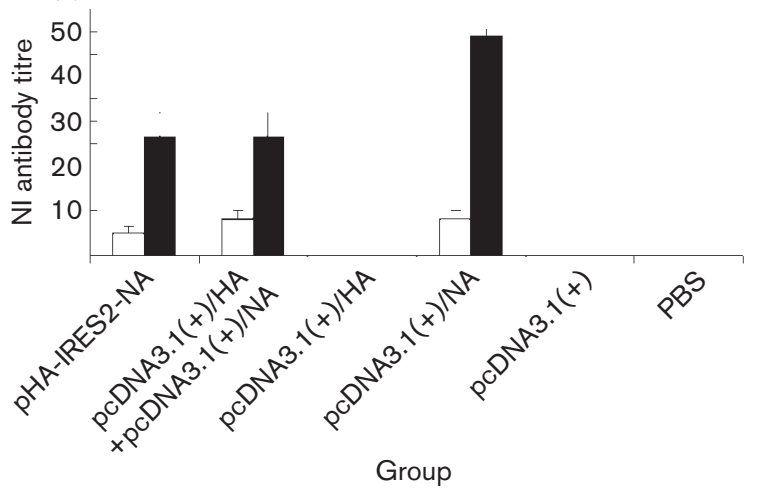

Fig. 4. Antibody titres obtained by the $\mathrm{HI}$ and $\mathrm{NI}$ assays. The mice were immunized twice with a 3 week interval. Sera samples were collected by tail bleed and heart bleed. (a) $\mathrm{HI}$ antibody levels in the secondary response were twofold to threefold higher than those of the primary response. (b) $\mathrm{NI}$ antibody levels in the secondary response increased fivefold in comparison with the primary response. White bars, primary response; black bars, secondary response.

to the immunized groups with plasmid DNA encoding a targeted gene, no serum antibodies specific for HA and NA were detected in the two control groups.

\section{Co-expression and single-expression $H A$ and/or NA DNA immunization induced a polarized robust Th1 (T helper) cell and Tc1 (cytotoxic T) cell response}

in vivo experiments have shown that immunization with plasmid DNA co-expression of HA and NA is capable of inducing humoral immunity and protecting against homologous virus attack; we have further used FACS to analyse its effect on cell-mediated immunity. Mononuclear cells were obtained from the spleens of BALB/c mice 3 days after virus challenge. The percentage of $\mathrm{CD} 3{ }^{+} \mathrm{CD} 8^{-}$cells $(68.23 \% \pm 2.99)$ for the PBS control group was higher than for any of the plasmid DNA immunization groups (the percentage of the other groups was $<45 \%$ ). Conversely, the percentage of $\mathrm{CD}^{+}{ }^{+} \mathrm{CD} 8{ }^{+}$cells for the PBS control group $(31.77 \% \pm 2.99)$ was significantly lower than for the other groups (the percentage of the other groups was $>55 \%)$, especially in the pHA-IRES2-NA $(64.2 \% \pm 4.55)$ and the HA+NA DNA groups $(67.99 \% \pm 5.58)$ (Fig. $5 \mathrm{a}$ ). The highest ratio of IFN $-\gamma^{+} \mathrm{CD}^{+} \mathrm{CD}^{-}$(Th1)/IL- $4^{+}$ $\mathrm{CD}^{+}{ }^{+} \mathrm{CD}^{-}{ }^{-}$(Th2) cells was $3.95 \pm 1.25$ in pHA-IRES2NA immunized mice, compared to pcDNA3.1 $(+) / \mathrm{HA}$ $(1.32 \pm 0.49)$, pcDNA3.1 $(+) / \mathrm{NA}(1.71 \pm 0.10)$, the mixture of pcDNA3.1 $(+) / \mathrm{HA}$ and pcDNA3.1 $(+) / \mathrm{NA} \quad(2.21 \pm$ $0.21), \quad$ pcDNA3.1 $(+) \quad(0.83 \pm 0.03)$ and PBS control $(0.46 \pm 0.04) \quad(P<0.05)$. The statistical analysis further indicated the ratio of $\mathrm{IFN}-\gamma^{+} \mathrm{CD} 3^{+} \mathrm{CD} 8^{-} / \mathrm{IL}-4^{+} \mathrm{CD} 3^{+}$ $\mathrm{CD}^{-}$cells in all of the plasmid immunized mice, including pcDNA3.1 $(+)$, exhibited a distinct difference in comparison with that of the PBS control $(P<0.05)$ (Fig. $5 b)$. The percentage of IFN- $\gamma^{+} \mathrm{CD}^{+} \mathrm{CD}^{+}(\mathrm{Tc1})$ cells was highest in mice treated with pHA-IRES2-NA $(97.95 \% \pm 1.5)$, lowest in mice treated with PBS $(46 \% \pm 0.5)$, and intermediate in mice treated with pcDNA3.1 $(+)(67.45 \% \pm 3.55)$ and the other mice groups, with $P<0.05$ for the test of significance among the groups. In contrast, there was no significant difference of $\mathrm{IL}-4^{+} \mathrm{CD} 3^{+} \mathrm{CD} 8{ }^{+}$cell numbers in the tested groups $(P>0.05)$ (Fig. 5c).

\section{Cellular response ex vivo induced by specific HA and NA antigen}

As shown in Fig. 6, the high degree of proliferation correlated by IFN- $\gamma$ production after specific HA and NA stimulation. The ratio of IFN- $\gamma^{+} \mathrm{CD} 3^{+} \mathrm{CD} 8^{-}$cells/IL- $4^{+}$ $\mathrm{CD} 3^{+} \mathrm{CD}^{-}$from $\mathrm{HA}$ and $\mathrm{NA}$ immunized mice was twofold-threefold higher compared to their respective controls after incubation with HA or NA antigen.

\section{DISCUSSION}

Recent studies of influenza virus DNA vaccines have focused on the influenza A virus. Previous studies from Robinson et al. (1993) and Ulmer et al. (1993) have sufficiently demonstrated the crucial role of HA- and NAencoding genes in providing protection and inducing an immune response, and identified them as potent candidate genes for influenza DNA vaccine, which has established a foundation for exploring influenza DNA vaccines. HA, the surface glycoprotein of influenza virus, has been shown to be the primary target for neutralizing antibodies and an essential determinant for pathogenicity, which plays the pivotal role in virus adsorption and membrane fusion (Chen et al., 1998; Wilson et al., 1981). The HA protein contains the cellular receptor-binding site with many conserved residues that make attractive targets for the design of vaccines and drugs to halt the progression of the virus (Wilson \& Itzstein, 2003). In comparison with HA, NA is relatively conserved, which stimulates the production of antibody equally. NA prevents viral self-aggregation and facilitates the release of virions from infected cells by cleaving sialic acid from the surface of infected cells and 

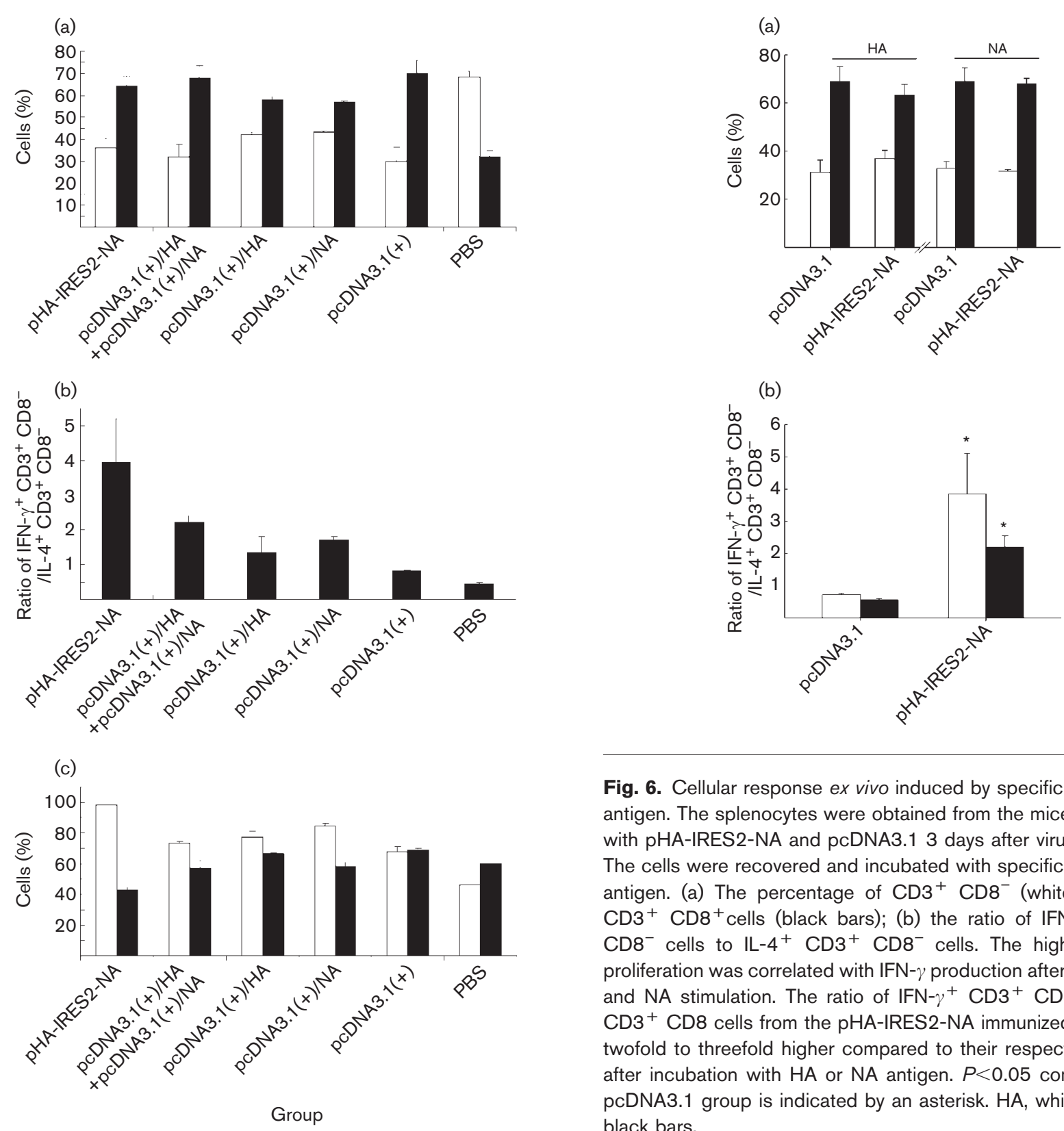

Fig. 6. Cellular response ex vivo induced by specific $H A$ and NA antigen. The splenocytes were obtained from the mice vaccinated with pHA-IRES2-NA and pcDNA3.1 3 days after virus challenge. The cells were recovered and incubated with specific $\mathrm{HA}$ and NA antigen. (a) The percentage of $\mathrm{CD}^{+} \mathrm{CD}^{-}$(white bars) and $\mathrm{CD}^{+}{ }^{+} \mathrm{CD} 8^{+}$cells (black bars); (b) the ratio of $\mathrm{IFN}-\gamma^{+} \mathrm{CD} 3^{+}$ $\mathrm{CD}^{-}$cells to $\mathrm{IL}_{-} 4^{+} \mathrm{CD} 3^{+} \mathrm{CD}^{-}$cells. The high degree of proliferation was correlated with IFN- $\gamma$ production after specific $\mathrm{HA}$ and NA stimulation. The ratio of $\mathrm{IFN}-\gamma^{+} \mathrm{CD} 3^{+} \mathrm{CD}^{-}$to $\mathrm{IL}-4^{+}$ $\mathrm{CD}^{+}$CD8 cells from the pHA-IRES2-NA immunized group was twofold to threefold higher compared to their respective controls after incubation with $\mathrm{HA}$ or NA antigen. $P<0.05$ compared with pcDNA3.1 group is indicated by an asterisk. HA, white bars; NA, black bars.

Fig. 5. T cell changes in the spleens of the tested mice. The mice were divided into six groups and inoculated with pHA-IRES2-NA, pcDNA3.1 $(+) / \mathrm{HA}$, pcDNA3.1(+)/NA, a mixture of pcDNA3.1 $(+) /$ $\mathrm{HA}$ and pcDNA3.1(+)/NA, pcDNA3.1 $(+)$ or PBS. Spleens were obtained 10 days after the virus challenge and spleen cells were used for CD3 and CD8 identification and analysis. (a) The percentage of $\mathrm{CD}^{+}{ }^{+} \mathrm{CD}^{-}$(white bars) and $\mathrm{CD}^{+}{ }^{+} \mathrm{CD} 8^{+}$cells (black bars); (b) the ratio of IFN- $\gamma^{+} \mathrm{CD}^{+} \mathrm{CD} 8^{-}$cells to $\mathrm{IL}-4^{+}$ $\mathrm{CD}^{+} \mathrm{CD}^{-}$cells; (c) the percentage of $\mathrm{IFN}-\gamma^{+} \mathrm{CD}^{+} \mathrm{CD}^{+}$ (white bars) and IL-4 ${ }^{+} \mathrm{CD} 3^{+} \mathrm{CD} 8^{+}$cells (black bars).

virus particles (Palese et al., 1974). NA has been thought of as a suitable target for designing agents against influenza viruses (Alymova et al., 2005).

Although the functional mechanism of the DNA vaccine is not well defined to this day, it is the prevalent opinion that the inoculation of naked plasmid DNA elicits robust and persistent antigen-specific antibody, Th cell and cytotoxic T lymphocyte (CTL) responses via intramuscular injection or gene-gun delivery. The method of DNA immunization has an influence on the pattern of type 1 and type 2 cytokines that can be induced in vivo for antigen-specific $\mathrm{CD}^{+}$and $\mathrm{CD}^{+} \mathrm{T}$ cells (Oran et al., 2003; Carter \& Dutton, 1996). IL-4 is regarded as a signature cytokine for a type $2 \mathrm{~T}$ cell response, and IFN- $\gamma$ as the signature cytokine for a type 1 response. $\mathrm{CD}^{+} \mathrm{T}$ cells are differentiated into two subsets, Th1 and Th2 cells, which secrete corresponding different cytokines - type 1 and type 
2 cytokines. Injection with plasmid DNAs dissolved in PBS (also called saline DNA immunization) into muscles has been shown to elicit a predominant Th1 response characterized by higher frequencies of IFN- $\gamma$ (Feltquate et al., 1997; Pertmer et al., 1996). The murine model experiment further revealed the signature cytokines that were accompanied by different predominant isotypes of antigen-specific antibody, for example, IFN- $\gamma$-producing $\mathrm{CD} 4{ }^{+} \mathrm{T}$ cells were associated with IgG2a generation, whereas IL-4-producing $\mathrm{CD} 4{ }^{+} \mathrm{T}$ cells biased the association with IgG1 generation (Snapper \& Paul, 1987). In the same way, two effector phenotypes of $\mathrm{CD} 8^{+} \mathrm{Tc}$ cells are distinguished by signature cytokines. IFN $-\gamma$ of type 1 cytokine is considered as a representative cytokine for Tc1, while IL-4, IL-10 and IL-5 of type 2 cytokines are representative for Tc2. Saline DNA immunization into muscles also activated Tc1 followed by a high magnitude of IFN- $\gamma$ level (Oran et al., 2003). In influenza infection models, higher numbers of $\mathrm{CD} 8^{+} \mathrm{T}$ cells were detected at an earlier time post-infection than $\mathrm{CD}^{+} \mathrm{T}$ cells, and $\mathrm{CD}^{+} \mathrm{T}$ cells expressed IFN $-\gamma$ upon restimulation ( $\mathrm{Sad}$ et al., 1995). In this study, the percentage of $\mathrm{CD}^{+} \mathrm{T}$ cells and IFN- $\gamma$-producing $\mathrm{CD} 8^{+}$cells, and the ratio of Th1 and Th2 cells increased to some extent in pHA-IRES2-NA, the mixture of $H A$ and NA DNA, and the single-expression $H A$ or NA DNA immunized mice compared to the control injected with PBS. The results confirmed the conclusion that saline DNA immunization raised strong Th1 response and stimulated CTL cell activation. Our findings further demonstrated that in addition to the essential role of $H A$ DNA as a part of the DNA vaccine preventing influenza virus, NA DNA also took a noticeable role in reducing virus replication in the lungs and providing sufficient protection against influenza virus. A slight increase in the percentage of $\mathrm{CD}^{+} \mathrm{T}$ cells and IFN- $\gamma$-producing $\mathrm{CD} 8^{+}$ cells, and the ratio of Th1 and Th2 cells in singleexpression NA DNA immunized mice suggested an involvement of NA in the cell-mediated response similar to the HA molecule. Our study showed that the coadministration of both $H A$ and NA DNAs induced a more enhanced protection against influenza challenge, which was consistent with the results from other studies (Zhang et al., 2005). Moreover, in our study we observed that coadministration of both $H A$ and NA DNAs effectively stimulated a cell-mediated response, which consequently increased the percentage of $\mathrm{CD}^{+} \mathrm{T}$ cells and IFN- $\gamma$ producing $\mathrm{CD}^{+}$cells, and the Th1 to Th2 ratio. More inspiringly, the novel co-expression plasmid pHA-IRES2NA exhibited a stronger activity in IFN- $\gamma$-producing $\mathrm{CD} 8^{+}$ cell activation than that of the mixture of pcDNA3.1 $(+) /$ HA and pcDNA3.1(+)/NA. Of course, we should take into account the function of the immunostimulatory sequence (ISS) carried by the vector in the immune response in addition to the effect of the targeted gene itself in the cellular response. This may be the rational explanation for why pcDNA3.1 could lead to the delicate rise in the percentage of $\mathrm{CD}^{+} \mathrm{T}$ cells and the ratio of Th1 and Th2 cells. The complicated mechanism of cellular response regulation induced by $H A$ and NA DNA vaccine could not be elucidated clearly and needs to be further explored step by step.

The utilization of plasmid DNA encoding influenza viral proteins to inoculate animals constitutes a promising alternative to conventional vaccination strategies. DNA vaccine offers many potential benefits, especially the efficacy of inducing a humoral immune response mimicking natural infection. To date, studies have detailed that inoculation of plasmid DNA encoding HA of influenza virus could elicit an immune response and generate an HA-specific neutralizing antibody, hence providing protection against influenza virus and even lasting for 1.5 years (Bot et al., 1997). There was evidence that inoculation of plasmid DNA encoding influenza virus NA could elicit the generation of a specific anti-NA antibody for inhibiting virus replication, alleviating the clinical symptoms and controlling the disease ( $\mathrm{Li}$ et al., 2006; Johansson et al., 1993). The immunization of plasmid DNA carrying $H A$ and/or NA in our experiment successfully induced the humoral response. The specific anti-HA level in co-expression HA and NA immunized mice was similar to the level in mice expressing HA- or NA-encoding DNA alone and mice expressing a mixture of HA + NA DNA, but the anti-NA level in the HA + NA DNA mice was lower than those other groups. This didn't exclude the unbalanced expression of $H A$ and $N A$ genes due to the insertion site of $N A$ gene downstream of the IRES sequence. Despite the insufficiencies, the novel $H A$ and NA DNA co-expression vaccine provided the most protection against the influenza virus, along with the use of a mixture of $H A$ and NA DNA.

In the field of influenza vaccine exploration, the researcher is facing another obstacle. There is a pre-existing immunity against influenza virus in the population due to prior infection or vaccination (He et al., 2003, 2006; Sasaki et al., 2007), which can function as protection against the same strain attack. However, it is well known that influenza virus has the distinct character of antigenic drift, which is the result of accumulation of point mutations in $H A$ and NA (Plotkin et al., 2002; McDonald et al., 2007). So that an individual who was infected by or vaccinated against a previously circulating influenza viruses may be susceptible to a new virus strain. However, the pre-existing immunity hampers the induction of a CTL response (de Mare et al., 2008). Therefore, the recombinant DNA vaccine from antigenic epitopes of HA and NA is a new protection strategy for influenza virus prevention. The development of effective vaccines, international surveillance and vaccine reformulation should not be overlooked at present and in the future.

In summary, the combination of $H A$ and NA DNA is mutually beneficial in producing the optimum efficiency for preventing influenza infection, and resulted in balanced and broadened immune responses. Both proteins expressed by HA and NA co-expression DNA vaccine can be detected in vivo and in vitro. More importantly, the vaccine can actively stimulate humoral and cell-mediated immune responses, and achieve the optimal protection against 
influenza infection. The immunization of this co-expression vector is enough to substitute for the mixture of $H A$ and NA DNA. Multi-gene expression possesses a significantly useful value in the field of biology. Using multi-genes of antigenic proteins or together with immunostimulatory molecules, and/or adjuvant or leader sequences, allows high-frequency transduction and makes possible the engineering of antigen-specific immune responses (Larregina \& Falo, 2000; Licht \& Peschel, 2002). The novel co-expression of both HA and NA may be a powerful approach in the development of the influenza DNA vaccine.

\section{ACKNOWLEDGEMENTS}

This work is supported by project grants from the National Natural Sciences Foundation of China and China Medical Board of Sichuan University.

\section{REFERENCES}

Alheim, M., Lazdina, U., Milich, D. R. \& Sallberg, M. (2001). Flow cytometric determination of cytokine production and proliferation in hepatitis B core antigen specific murine CD4 cells: lack of correlation between number of cytokine producing cells and cytokine levels in supernatant. J Immunol Methods 258, 157-167.

Alymova, I. V., Taylor, G. \& Portner, A. (2005). Neuraminidase inhibitors as antiviral agents. Curr Drug Targets Infect Disord 5, 401409.

Aymard-Henry, M., Coleman, M. T., Dowdle, W. R., Laver, W. G., Schild, G. C. \& Webster, R. G. (1973). Influenzavirus neuraminidase and neuraminidase-inhibition test procedures. Bull World Health Organ 48, 199-202.

Bona, C., Radu, D. \& Kodera, T. (2004). Molecular studies on the diversification of haemagglutinin-specific human neonatal repertoire subsequent to immunization with naked DNA. Vaccine 22, 16241630.

Bot, A., Antohi, S., Bot, S., Garcia-Sastre, A. \& Bona, C. (1997). Induction of humoral and cellular immunity against influenza virus by immunization of newborn mice with a plasmid bearing a hemagglutinin gene. Int Immunol 9, 1641-1650.

Carter, L. L. \& Dutton, R. W. (1996). Type 1 and type 2: a fundamental dichotomy for all T-cell subsets. Curr Opin Immunol 8, 336-342.

Chen, J., Lee, K. H., Steinhauer, D. A., Stevens, D. J., Skehel, J. J. \& Wiley, D. C. (1998). Structure of the hemagglutinin precursor cleavage site, a determinant of influenza pathogenicity and the origin of the labile conformation. Cell 95, 409-417.

Chen, J., Fang, F., Li, X., Chang, H. \& Chen, Z. (2005). Protection against influenza virus infection in BALB/c mice immunized with a single dose of neuraminidase-expressing DNAs by electroporation. Vaccine 23, 4322-4328.

De Mare, A., Bungener, L. B., Regts, J., de Vries-Idema, J., Van der Zee, A. G., Wilschut, J. \& Daemen, T. (2008). The effect of pre-existing immunity on the capacity of influenza virosomes to induce cytotoxic T lymphocyte activity. Vaccine 26, 2314-2321.

Donnelly, J. J., Ulmer, J. B., Shiver, J. W. \& Liu, M. A. (1997). DNA vaccine. Annu Rev Immunol 15, 617-648.

Feltquate, D. M., Heaney, S., Webster, R. G. \& Robinson, H. L. (1997). Different $T$ helper cell types and antibody isotypes generated by saline and gene gun DNA immunization. J Immunol 158, 2278-2284.
Fiers, W., Neirynck, S., Deroo, T., Saelens, X. \& Jou, W. M. (2001). Soluble recombinant influenza vaccines. Philos Trans $R$ Soc Lond $B$ Biol Sci 356, 1961-1963.

Gruber, W. C. (2002). The role of live influenza vaccines in children. Vaccine 20, S66-S73.

He, X. S., Mahmood, K., Maecker, H. T., Holmes, T. H., Kemble, G. W., Arvin, A. M. \& Greenberg, H. B. (2003). Analysis of the frequencies and of the memory $\mathrm{T}$ cell phenotypes of human $\mathrm{CD} 8^{+} \mathrm{T}$ cells specific for influenza A viruses. J Infect Dis 187, 1075-1084.

He, X. S., Holmes, T. H., Zhang, C., Mahmood, K., Kemble, G. W., Lewis, D. B., Dekker, C. L., Greenberg, H. B. \& Arvin, A. M. (2006). Cellular immune responses in children and adults receiving inactivated or live attenuated influenza vaccines. J Virol 80, 11756-11766.

Johansson, B. E., Grajower, B. \& Kilbourne, E. D. (1993). Infectionpermissive immunization with influenza virus neuraminidase prevents weight loss in infected mice. Vaccine 11, 1037-1039.

Johnson, P. A., Conway, M. A., Daly, J., Nicolson, C., Robertson, J. \& Mills, K. H. (2000). Plasmid DNA encoding influenza virus haemagglutinin induces Th1 cells and protection against respiratory infection despite its limited ability to generate antibody responses. J Gen Virol 81, 1737-1745.

Lambkin, R., Novelli, P., Oxford, J. \& Gelder, C. (2004). Human genetics and responses to influenza vaccination: clinical implication. Am J Pharmacogenomics 4, 293-298.

Larregina, A. T. \& Falo, L. D., Jr (2000). Generating and regulating immune responses through cutaneous gene delivery. Hum Gene Ther 11, 2301-2305.

Li, X., Fang, F., Song, Y., Yan, H., Chang, H., Sun, S. \& Chen, Z. (2006). Essential sequence of influenza neuraminidase DNA to provide protection against lethal viral infection. DNA Cell Biol 25, 197-205.

Licht, T. \& Peschel, C. (2002). Restoration of transgene expression in hematopoietic cells with drug-selectable marker genes. Curr Gene Ther 2, 227-234.

McDonald, N. J., Smith, C. B. \& Cox, N. J. (2007). Antigenic drift in the evolution of H1N1 influenza A viruses resulting from deletion of a single amino acid in the haemagglutinin gene. J Gen Virol 88, 32093213.

Oran, A. E. \& Robinson, H. L. (2003). DNA vaccines, combining form of antigen and method of delivery to raise a spectrum of IFN- $\gamma$ and IL-4-producing CD4 ${ }^{+}$and CD8 ${ }^{+}$T cells. J Immunol 171, 1999-2005.

Palese, P., Tobita, K., Ueda, M. \& Compans, R. W. (1974). Characterization of temperature-sensitive influenza virus mutants defective in neuraminidase. Virology 61, 397-410.

Pertmer, T. M., Roberts, T. R. \& Haynes, J. R. (1996). Influenza virus nucleoprotein-specific immunoglobulin G subclass and cytokine responses elicited by DNA vaccination are dependent on the route of vector DNA delivery. J Virol 70, 6119-6125.

Plotkin, J. B., Dushoff, J. \& Levin, S. A. (2002). Hemagglutinin sequence clusters and the antigenic evolution of influenza $A$ virus. Proc Natl Acad Sci U S A 99, 6263-6268.

Reed, L. J. \& Muench, H. (1938). A simple method of estimating fifty percent endpoints. Am J Hyg 27, 493-497.

Robinson, H. L., Hunt, L. A. \& Webster, R. G. (1992). Protection against a lethal influenza virus challenge by immunization with a haemagglutinin-expressing plasmid DNA. Vaccine 11, 957-960.

Sad, S., Marcotte, R. \& Mosmann, T. R. (1995). Cytokine-induced differentiation of precursor mouse $\mathrm{CD} 8^{+} \mathrm{T}$ cells into cytotoxic $\mathrm{CD} 8^{+}$ $\mathrm{T}$ cells secreting Th1 or Th2 cytokines. Immunity 2, 271-279.

Sasaki, S., Jaimes, M. C., Holmes, T. H., Dekker, C. L., Mahmood, K., Kemble, G. W., Arvin, A. M. \& Greenberg, H. B. (2007). Comparison 
of the influenza virus-specific effector and memory B-cell responses to immunization of children and adults with live attenuated or inactivated influenza virus vaccines. J Virol 81, 215-228.

Shen, Y., Muramatsu, S. I., Ikeguchi, K., Fujimoto, K. I., Fan, D. S., Ogawa, M., Mizukami, H., Urabe, M., Kume, A. \& other authors (2000). Triple transduction with adeno-associated virus vectors expressing tyrosine hydroxylase, aromatic-L-amino-acid decarboxylase, and GTP cyclohydrolase I for gene therapy of Parkinson's disease. Hum Gene Ther 11, 1509-1519.

Snapper, C. M. \& Paul, W. E. (1987). Interferon- $\gamma$ and B cell stimulatory factor-1 reciprocally regulate Ig isotype production. Science 236, 944-947.

Tamura, S., Ito, Y., Asanuma, H., Hirabayashi, Y., Suzuki, Y., Nagamine, T., Aizawa, C. \& Kurata, T. (1992a). Cross-protection against influenza virus infection afforded by trivalent inactivated vaccines inoculated intranasally with cholera toxin B subunit. J Immunol 149, 981-988.

Tamura, S. I., Asanuma, H., Ito, Y., Hirabayashi, Y., Suzuki, Y., Nagamine, T., Aizawa, C., Kurata, T. \& Oya, A. (1992b). Superior cross-protective effect of nasal vaccination to subcutaneous inoculation with influenza hemagglutinin vaccine. Eur J Immunol 22, 477481.
Tighe, H., Corr, M., Roman, M. \& Raz, E. (1998). Gene vaccination: plasmid DNA is more than just a blueprint. Immunol Today 19, 8997.

Ulmer, J. B. (2002). Influenza DNA vaccines. Vaccine 20, S74-S76.

Ulmer, J. B., Donnelly, J. J., Paker, S. E., Rhodes, G. H., Felgner, P. L., Dwarki, V. J., Gromkowski, S. H., Deck, R. R., DeWitt, C. M. \& other authors (1993). Heterologous protection against influenza by infection of DNA encoding a viral protein. Science 259, 1745-1749.

Wilson, J. C. \& Itzstein, M. (2003). Recent strategies in the search for new anti-influenza therapies. Curr Drug Targets 4, 389-408.

Wilson, I. A., Skehel, J. J. \& Wiley, D. C. (1981). Structure of the haemagglutinin membrane glycoprotein of influenza virus at $3 \AA$ resolution. Nature 289, 366-373.

Zhang, F., Chen, J., Fang, F., Zhou, Y., Wu, J., Chang, H., Zhang, R., Wang, F., Li, X. \& other authors (2005). Maternal immunization with both hemagglutinin and neuraminidase expressing DNAs provides an enhanced protection against a lethal influenza virus challenge in infant and adult mice. DNA Cell Biol 24, 758-765.

Zhang, W., Li, M., Cao, K., Yang, J., Shi, Q., Wang, B., Jiang, Z. \& Li, H. (2006). Construction of eukaryotic expressing plasmids encoding $H A$ and $H A_{1}$ of influenza $\mathrm{A}$ virus and their transient expression in HEK293 cells. J Huazhong Univ Sci Technolog Med Sci 26, 225-230. 